\title{
A Finite Difference Scheme for Compressible Miscible Displacement Flow in Porous Media on Grids with Local Refinement in Time
}

\author{
Wei Liu \\ School of Mathematic and Quantitative Economics, Shandong University of Finance and Economics, Jinan 250014, China \\ Correspondence should be addressed to Wei Liu; liuwei@sdufe.edu.cn
}

Received 19 October 2012; Accepted 19 December 2012

Academic Editor: Xinan Hao

Copyright (c) 2013 Wei Liu. This is an open access article distributed under the Creative Commons Attribution License, which permits unrestricted use, distribution, and reproduction in any medium, provided the original work is properly cited.

Considering two-dimensional compressible miscible displacement flow in porous media, finite difference schemes on grids with local refinement in time are constructed and studied. The construction utilizes a modified upwind approximation and linear interpolation at the slave nodes. Error analysis is presented in the maximum norm and numerical examples illustrating the theory are given.

\section{Introduction}

Numerical models of percolation flow are almost built up on a basis of the finite difference method to solve the system of partial differential equations. Usually, grids that we used are thinner, then the truncation error is smaller and the computing accuracy is higher. In order to assure certain computation accuracy, the grid number cannot be too little. But on the other hand, along with the increment of the grid number, the computation cost is greatly increased and the algebraic system which is formed finally cannot be resolved, even with the largest of today's supercomputers. Actually, we only need to refine grids around wells, cracks, obstacles, domain boundaries, and so forth, where the pressure changes radically. But because the finite difference grid is composed of straight lines and the grid density cannot be varied with space, it limits the simulating scale and the simulating accuracy. For the local grid refinement technique, we still make use of the finite difference grid system and divide partial grids which are needed to be refined into fine grids. In this way, we can resolve problems, such as small well spacing, fault, and boundary, and we can improve the simulating accuracy and extend the simulating scale [1]. Ewing et al. construct some finite difference approximations on grids with local refinement in space for the ellipse equation and obtain error estimates in the $H^{1}$-norm [2]. Cai et al. analyze stationary local grid refinement for the diffusion equation $[3,4]$. Ewing et al. derive implicit schemes on the basis of a finite volume approach by approximation of the balance equation. This approach leads to schemes that are locally conservative and are absolutely stable [5]. Ewing et al. construct and study finite difference schemes for transient convection-diffusion problems on grids with local refinement in time and space. The proposed schemes are unconditionally stable and use linear interpolation along the interface [6]. Respectively for incompressible miscible displacement flow in porous media and the semiconductor device problem, authors discuss discrete schemes, error estimates, and numerical examples on composite triangular grids $[7,8]$.

In this paper, we study a finite difference scheme on grids with local refinement in time for two-dimensional compressible miscible displacement flow in porous media. The pressure equation is approximated by a five-point difference scheme, and the saturation equation is discretized by a modified upwind scheme. At the slave nodes, the construction utilizes linear interpolation. Finally, error analysis in the maximum norm is derived and numerical examples are given to support the numerical method and its convergence.

The paper is organized as follows. In Sections 2 and 3, we formulate the problem and introduce the necessary notations. In Section 4 the construction of the finite difference scheme is presented. The error analysis is addressed in Section 5. 
Finally, in Section 6 we present numerical experiments that conform our theoretical results.

\section{Problem Formulation}

We will consider a system of three nonlinear partial differential equations in a bounded domain $\Omega \subset R^{2}$, which forms a basic model of compressible miscible displacement flow in porous media [9-11]:

(a) $\quad d(c) \frac{\partial p}{\partial t}+\nabla \cdot u=q(x, t), \quad x=\left(x_{1}, x_{2}\right) \in \Omega$,

$$
t \in J=[0, T],
$$

(b) $u=-a(c) \nabla p, \quad(x, t) \in \Omega \times J$,

(c) $\phi(x) \frac{\partial c}{\partial t}+b(c) \frac{\partial p}{\partial t}+u \cdot \nabla c-\nabla \cdot(D \nabla c)=f(x, t, c)$,

$$
(x, t) \in \Omega \times J,
$$

where

$$
\begin{gathered}
c=c_{1}=1-c_{2}, \\
a(c)=a(x, c)=\frac{k(x)}{\mu(c)}, \\
d(c)=d(x, c)=\phi(x) \sum_{j=1}^{2} Z_{j} c_{j},
\end{gathered}
$$

$c_{i}(i=1,2)$ is the saturation of the $i$ th component in mixed liquid, $Z_{j}$ is the $j$ th component of compression constant factor, $\phi$ is the porosity of the rock, $k$ is the permeability of the rock, $\mu$ is the viscosity of the fluid, $D=\phi(x) d_{m} I$ which is the $2 \times 2$ diffusion matrix, $d_{m}$ is the diffusion coefficient, and $I$ is the unit matrix. The unknowns are the pressure function $p(x, t)$ and the saturation function $c(x, t)$.

In addition, we have boundary conditions

$$
\begin{aligned}
& p=e(x, t), \quad x \in \partial \Omega, t \in J, \\
& c=h(x, t), \quad x \in \partial \Omega, t \in J,
\end{aligned}
$$

and initial conditions

$$
\begin{aligned}
& p(x, 0)=p_{0}(x), \quad x \in \Omega, \\
& c(x, 0)=c_{0}(x), \quad x \in \Omega,
\end{aligned}
$$

where $\Omega$ is a plane bounded domain and $\partial \Omega$ is the boundary of $\Omega$.

Usually this question is positive. Suppose the coefficients of (1) satisfy

$$
\begin{gathered}
0<a_{*} \leq a(c) \leq a^{*}, \\
0<d_{*} \leq d(c) \leq d^{*}, \\
0<D_{*} \leq D(x) \leq D^{*}, \\
\left|\frac{\partial a}{\partial c}(x, c)\right| \leq K^{*}
\end{gathered}
$$

where $a_{*}, a^{*}, d_{*}, d^{*}, D_{*}, D^{*}, K^{*}$ are positive constants and $d(c), b(c)$, and $f(c)$ are Lipschitz continuous in the $\varepsilon_{0}$ neighborhood of the solution.

We suppose that the exact solutions of (1) are distributed smoothly; $p$ and $c$ satisfy

$$
\begin{gathered}
p, c \in L^{\infty}\left(0, T ; W^{4, \infty}(\Omega)\right), \\
\frac{\partial^{2} p}{\partial t^{2}}, \frac{\partial^{2} c}{\partial t^{2}} \in L^{\infty}\left(0, T ; L^{\infty}(\Omega)\right) .
\end{gathered}
$$

Throughout this paper, the notations $K_{i}(i=0,1, \ldots, M)$ are used to denote generic constants.

\section{Grids, Grid Functions, and Associated Notations}

First, $\Omega=[0,1]^{2}$ is discretized using a regular grid with a parameter $h$. The spatial nodes of the grid on $\Omega$ are then defined by $x=\left(x_{1}, x_{2}\right)=\left(n_{1} h, n_{2} h\right)$, where $n_{1}=0, \ldots, N$, $n_{2}=0, \ldots, N, h=1 / N$. Next, we introduce closed domains $\left\{\Omega_{k}\right\}_{k=1}^{M}$, which are subsets of $\Omega$ with boundaries aligned with the spatial discretization already defined. Further, it is required that $\bigcup_{k=1}^{M} \Omega_{k} \subset \Omega$, and we set $\Omega_{0}=\Omega \backslash \bigcup_{k=1}^{M} \Omega_{k}$. In order to avoid unnecessary complications, for $i, j>0$, we assume that $\operatorname{dist}\left(\Omega_{i}, \Omega_{j}\right) \geq l h$, where $l>1$ is an integer.

With each subdomain $\Omega_{i}$, we associate corresponding sets of nodal points: $\omega_{i}$ is defined to be the set of all nodes of the discretization of $\Omega$ that are in $\Omega_{i}$. We require $\omega_{i} \cap \omega_{j}=\emptyset$, for $i \neq j, i, j=0, \ldots, M$. And assume that there is no spatial refinement. In each $\omega_{i}, i=0, \ldots, M$, we define a subset of boundary nodes $\gamma_{i}$ as the nodes which have at least one neighbor not in $\omega_{i}$. Then set $\omega=\bigcup_{i=0}^{M} \omega_{i}$.

A discrete time-step $\tau_{i}$ is associated with each $\Omega_{i}$ such that, for integers $m_{i}$,

$$
\tau_{0}=m_{i} \tau_{i}, \quad i=0, \ldots, M, \quad m_{0}=1 .
$$

Consequently, discrete time levels $t_{i}^{j}$ for $\Omega_{i}$ are defined by $t_{i}^{j}=$ $j \tau_{i}, j=1,2, \ldots,\left[T / \tau_{i}\right]$. Finally, we define the grid points $g$ by setting

$$
g_{i}=\bigcup_{\substack{x \in \omega_{i} \\ j=1,2, \ldots}}\left(x, j \tau_{i}\right), \quad i=0, \ldots, M, \quad g=\bigcup_{i=0}^{M} g_{i} .
$$

We continue by specifying the nodes in $g_{i}$ between time levels $t_{0}^{l}$ and $t_{0}^{l+1}$ as

$$
\begin{array}{r}
g_{i}^{l}=\bigcup_{\substack{x \in \omega_{i} \\
j=0}}^{m_{i}}\left(x, t_{0}^{l}+j \tau_{i}\right)=\bigcup_{\substack{x \in \omega_{i} \\
j=0}}^{m_{i}}\left(x, t_{i}^{l, j}\right), \\
t_{i}^{l, j}=t_{0}^{l}+j \tau_{i}, \quad i=0, \ldots, M .
\end{array}
$$

Correspondingly, the boundary nodes of $g_{i}^{l}$ are defined by

$$
\partial g_{i}^{l}=\bigcup_{\substack{x \in \gamma_{i} \\ j=0}}^{m_{i}}\left(x, t_{i}^{l, j}\right), \quad i=0, \ldots, M .
$$


The grid function $y(x, t)$ is a function defined at the grid points of $g$. we denote the nodal values of a grid function $y(x, t)$ between time levels $t_{0}^{l}$ and $t_{0}^{l+1}$ as

$$
y(x, t)=y\left(x_{1}, x_{2}, t_{i}^{l, j}\right)=y_{n_{1}, n_{2}}^{l, j},
$$

for $x \in \omega_{i}, i>0, j=0, \ldots, m_{i}$. For $x \in \omega_{0}$ we define

$$
y(x, t)=y\left(x_{1}, x_{2}, t_{0}^{l+1}\right)=y_{n_{1}, n_{2}}^{l+1} .
$$

$\delta_{x_{1}}, \delta_{\bar{x}_{1}}$ and $\delta_{x_{2}}, \delta_{\bar{x}_{2}}$ are the divided forward and backward difference operators, respectively, in $x_{1}$ and $x_{2}$ direction. Also define the divided backward time difference by

$$
\begin{array}{r}
\delta_{\tau_{0}} y_{0}^{l}(x)=\frac{y_{0}^{l}(x)-y_{0}^{l-1}(x)}{\tau_{0}}, \quad x \in \omega_{0}, \\
\delta_{\tau_{i}} y_{i}^{l, j}(x)=\frac{y_{i}^{l, j}(x)-y_{i}^{l, j-1}(x)}{\tau_{i}}, \quad x \in \omega_{i}, \\
j=1,2, \ldots, m_{i}, i=1, \ldots, M .
\end{array}
$$

\section{Construction of the Finite Difference Schemes}

Let $P, U$, and $C$ be the numerical approximations to the pressure $p$, the velocity $u$, and the saturation $c$, respectively. The approximation for the pressure and the concentration approximation are done on composite grids in time.

First for the pressure equation, we let

$$
\begin{aligned}
A_{0}^{l} & \left(x_{1}+\frac{h}{2}, x_{2}\right) \\
\quad & =\frac{1}{2}\left[a\left(x, C_{0}^{l}(x)\right)+a\left(x_{1}+h, x_{2}, C_{0}^{l}\left(x_{1}+h, x_{2}\right)\right)\right] .
\end{aligned}
$$

Similarly we define $A_{0}^{l}\left(x_{1}, x_{2}+h / 2\right)$, then let

$$
\begin{gathered}
\delta_{\bar{x}_{1}}\left(A_{0}^{l} \delta_{x_{1}} P_{0}^{l+1}\right)(x) \\
=h^{-2}\left[A_{0}^{l}\left(x_{1}+\frac{h}{2}, x_{2}\right)\left(P_{0}^{l+1}\left(x_{1}+h, x_{2}\right)-P_{0}^{l+1}(x)\right)\right. \\
\left.\quad-A_{0}^{l}\left(x_{1}-\frac{h}{2}, x_{2}\right)\left(P_{0}^{l+1}(x)-P_{0}^{l+1}\left(x_{1}-h, x_{2}\right)\right)\right], \\
\delta_{\bar{x}_{2}}\left(A_{0}^{l} \delta_{x_{2}} P_{0}^{l+1}\right)(x) \\
=h^{-2}\left[A_{0}^{l}\left(x_{1}, x_{2}+\frac{h}{2}\right)\left(P_{0}^{l+1}\left(x_{1}, x_{2}+h\right)-P_{0}^{l+1}(x)\right)\right. \\
\left.\quad-A_{0}^{l}\left(x_{1}, x_{2}-\frac{h}{2}\right)\left(P_{0}^{l+1}(x)-P_{0}^{l+1}\left(x_{1}, x_{2}-h\right)\right)\right], \\
\nabla_{h}\left(A_{0}^{l} \nabla_{h} P_{0}^{l+1}\right)(x)=\delta_{\bar{x}_{1}}\left(A_{0}^{l} \delta_{x_{1}} P_{0}^{l+1}\right)(x) \\
\quad+\delta_{\bar{x}_{2}}\left(A_{0}^{l} \delta_{x_{2}} P_{0}^{l+1}\right)(x) .
\end{gathered}
$$

is

For regular coarse grids, the five-point difference scheme

$$
d\left(C_{0}^{l}(x)\right) \delta_{\tau_{0}} P_{0}^{l+1}(x)-L_{h}^{p} P_{0}^{l+1}(x)=q_{0}^{l+1}(x), \quad x \in g_{0}^{l},
$$

where the difference operator $L_{h}^{p} P_{0}^{l+1}(x)=-\nabla_{h}\left(A_{0}^{l} \nabla_{h} P_{0}^{l+1}\right)$ $(x)$. The Darcy velocity $U^{l}=\left(U_{1,0}^{l}, U_{2,0}^{l}\right)$ is computed as follows:

$$
\begin{aligned}
U_{1,0}^{l}(x)=-\frac{1}{2}[ & A_{0}^{l}\left(x_{1}+\frac{h}{2}, x_{2}\right) \delta_{x_{1}} P_{0}^{l+1}(x) \\
& \left.+A_{0}^{l}\left(x_{1}-\frac{h}{2}, x_{2}\right) \delta_{\bar{x}_{1}} P_{0}^{l+1}(x)\right] .
\end{aligned}
$$

$U_{2,0}^{l}$ corresponds to another direction, and the computational formula is similar to $U_{1,0}^{l}$.

Next we consider the saturation equation (1)(c). The positive and negative of the function $v$ are defined as $v^{+}=$ $(1 / 2)(v+|v|) \geq 0$ and $v^{-}=(1 / 2)(v-|v|) \leq 0$. For regular coarse grids, the upwind difference scheme of the saturation equation is

$$
\begin{aligned}
& \phi(x) \delta_{\tau_{0}} C_{0}^{l+1}(x)-L_{h}^{c} C_{0}^{l+1}(x) \\
& \quad=f\left(x, t_{0}^{l}, C_{0}^{l}(x)\right)-b\left(C_{0}^{l}(x)\right) \delta_{\tau_{0}} P_{0}^{l+1}(x), \quad x \in g_{0}^{l},
\end{aligned}
$$

where

$$
\begin{aligned}
& L_{h}^{c} C_{0}^{l+1}(x)=\left(1+\frac{h}{2}\left|U_{1,0}^{l}\right| D^{-1}\right)^{-1} \delta_{\bar{x}_{1}}\left(D \delta_{x_{1}} C_{0}^{l+1}\right)(x) \\
& +\left(1+\frac{h}{2}\left|U_{2,0}^{l}\right| D^{-1}\right)^{-1} \delta_{\bar{x}_{2}}\left(D \delta_{x_{2}} C_{0}^{l+1}\right)(x) \\
& -\delta_{U_{1,0}^{l}, x_{1}} C^{l+1}(x)-\delta_{U_{2,0}^{l}, x_{2}} C^{l+1}(x), \\
& \delta_{U_{1,0}^{l}, x_{1}} C_{0}^{l+1}(x) \\
& =U_{1,0}^{l}(x) \\
& \times\left\{H\left(U_{1,0}^{l}(x)\right) D^{-1} D_{x_{1}-h / 2, x_{2}} \delta_{\bar{x}_{1}} C_{0}^{l+1}(x)\right. \\
& \left.+\left(1-H\left(U_{1,0}^{l}(x)\right)\right) D^{-1} D_{x_{1}+h / 2, x_{2}} \delta_{x_{1}} C_{0}^{l+1}(x)\right\}, \\
& \delta_{U_{2,0}^{l}, x_{2}} C_{0}^{l+1}(x) \\
& =U_{2,0}^{l}(x) \\
& \times\left\{H\left(U_{2,0}^{l}(x)\right) D^{-1} D_{x_{1}, x_{2}-h / 2} \delta_{\bar{x}_{2}} C_{0}^{l+1}(x)\right. \\
& \left.+\left(1-H\left(U_{2,0}^{l}(x)\right)\right) D^{-1} D_{x_{1}, x_{2}+h / 2} \delta_{x_{2}} C_{0}^{l+1}(x)\right\}, \\
& H(z)= \begin{cases}1, & z \geq 0, \\
0, & z<0 .\end{cases}
\end{aligned}
$$




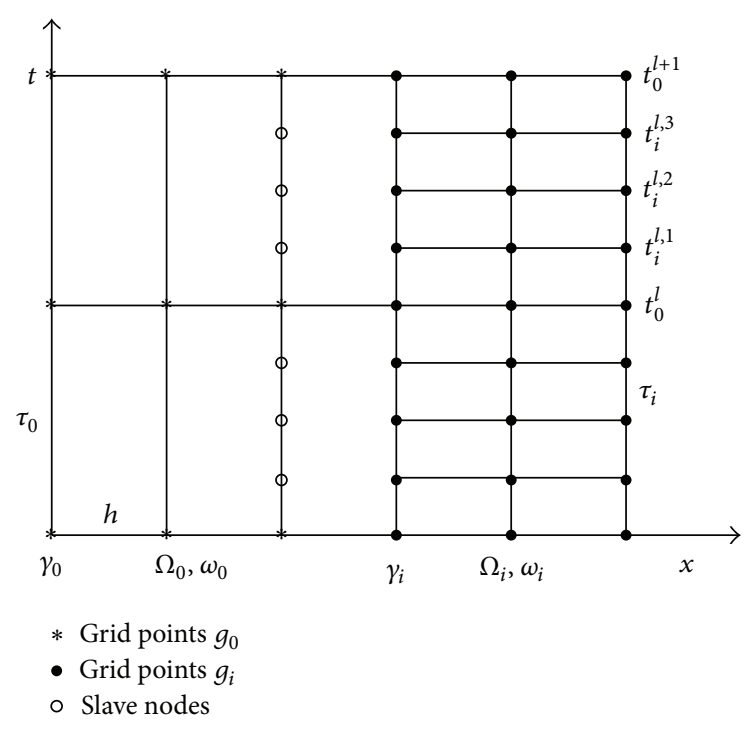

FIgURE 1: Grid with local refinement in time.

In the region that is refined in time, we can construct finite difference schemes similar to (16)-(18). It is obvious that at time $t_{i}^{l, j}=l \tau_{0}+j \tau_{i}, j=1, \ldots, m_{i}$, when the difference operators defined above are applied to the points of $\gamma_{i}$, not all space-time positions required correspond to actual nodes in $g$. For such cases, we define

$$
\begin{aligned}
& P\left(x, t_{i}^{l, j}\right)=\frac{j}{m_{i}} P\left(x, t_{0}^{l+1}\right)+\frac{m_{i}-j}{m_{i}} P\left(x, t_{0}^{l}\right), \\
& C\left(x, t_{i}^{l, j}\right)=\frac{j}{m_{i}} C\left(x, t_{0}^{l+1}\right)+\frac{m_{i}-j}{m_{i}} C\left(x, t_{0}^{l}\right) .
\end{aligned}
$$

In Figure 1, the slave nodes represent the missing space-time positions in the stencil of nodes in $\partial q_{i}^{l}, i>0$. The values there are computed by the interpolation formula (20).

The discretization schemes of (1)-(4) on composite grids are given by

$$
\begin{gathered}
d\left(C_{0}^{l}(x)\right) \delta_{\tau_{0}} P_{0}^{l+1}(x)-L_{h}^{p} P_{0}^{l+1}(x)=q_{0}^{l+1}(x), \quad x \in g_{0}^{l}, \\
d\left(C_{i}^{l, j}(x)\right) \delta_{\tau_{i}} P_{i}^{l, j+1}(x)-L_{h}^{p} P_{i}^{l, j+1}(x)=q_{i}^{l, j+1}(x), \\
x \in g_{i}^{l}, i=1, \ldots, M, \\
P(x, t)=e(x, t), \quad x \in \partial \Omega,
\end{gathered}
$$

$$
\begin{aligned}
& U_{1,0}^{l}(x) \\
& \begin{array}{l}
=-\frac{1}{2}\left[A_{0}^{l}\left(x_{1}+\frac{h}{2}, x_{2}\right) \partial_{x_{1}} P_{0}^{l+1}(x)\right. \\
\left.\quad+A_{0}^{l}\left(x_{1}-\frac{h}{2}, x_{2}\right) \partial_{\bar{x}_{1}} P_{0}^{l+1}(x)\right], \quad x \in g_{0}^{l},
\end{array}
\end{aligned}
$$

$$
\begin{aligned}
& U_{1, i}^{l, j}(x) \\
& =-\frac{1}{2}\left[A_{i}^{l, j}\left(x_{1}+\frac{h}{2}, x_{2}\right) \partial_{x_{1}} P_{i}^{l, j+1}(x)\right. \\
& \left.+A_{i}^{l, j}\left(x_{1}-\frac{h}{2}, x_{2}\right) \partial_{\bar{x}_{1}} P_{i}^{l, j+1}(x)\right], \\
& x \in g_{i}^{l}, i=1, \ldots, M, m=1,2 .
\end{aligned}
$$

$U_{2,0}^{l}, U_{2, i}^{l, j}$ correspond to another direction, and computational formulae are similar to (22).

$$
\begin{aligned}
\phi(x) & \delta_{\tau_{0}} C_{0}^{l+1}(x)-L_{h}^{c} C_{0}^{l+1}(x) \\
= & f\left(x, t_{0}^{l}, C_{0}^{l}(x)\right) \\
& -b\left(C^{l}(x)\right) \delta_{\tau_{0}} P_{0}^{l+1}(x), \quad x \in g_{0}^{l},
\end{aligned}
$$

$$
\begin{gathered}
\phi(x) \delta_{\tau_{i}} C_{i}^{l, j+1}(x)-L_{h}^{c} C_{i}^{l, j+1}(x) \\
=f\left(x, t_{i}^{l, j}, C_{i}^{l, j}(x)\right) \\
-b\left(C_{i}^{l, j}(x)\right) \delta_{\tau_{0}} P_{i}^{l, j+1}(x), \quad x \in g_{i}^{l}, i=1, \ldots, M, \\
C(x, t)=h(x, t), \quad x \in \partial \Omega .
\end{gathered}
$$

\section{Error Analysis}

The discrete inner product and $L^{2}$-norm of grid functions are defined, respectively, by

$$
\begin{gathered}
(y, v)=\sum_{x \in \omega} h^{2} y(x) v(x), \\
\|y\|_{0, \omega}=(y, y)^{1 / 2} .
\end{gathered}
$$

We also use the standard notation for the discrete $H^{1}$-norm of a grid function in the Sobolev space $H_{0}^{1}(\omega)$ :

$$
\|y\|_{1, \omega}^{2}=\|y\|_{0, \omega}^{2}+\sum_{i=1}^{2}\left\|\delta_{\bar{x}_{i}} y\right\|_{0, \omega}^{2} .
$$

Define the error of the above scheme by

$$
\begin{gathered}
\pi_{0}^{l}(x)=p_{0}^{l}(x)-P_{0}^{l}(x), \\
\xi_{0}^{l}(x)=c_{0}^{l}(x)-C_{0}^{l}(x), \quad x \in \omega_{0}, \\
\pi_{i}^{l, j}(x)=p_{i}^{l, j}(x)-P_{i}^{l, j}(x), \\
\xi_{i}^{l, j}(x)=c_{i}^{l, j}(x)-C_{i}^{l, j}(x), \\
x \in \omega_{i}, i=1, \ldots, M .
\end{gathered}
$$


Firstly consider the pressure equation. Using (1)(a) and (21), we get the error equation:

(a) $\pi(x, t)=0, \quad x \in \partial \Omega$,

(b) $\quad d\left(C_{0}^{l}(x)\right) \delta_{\tau_{0}} \pi_{0}^{l+1}(x)-L_{h}^{p} \pi_{0}^{l+1}(x)$

$$
=K_{0}\left(h^{2}+\tau_{0}+\xi_{0}^{l}(x)\right), \quad x \in g_{0}^{l},
$$

(c) $\quad d\left(C_{i}^{l, j}(x)\right) \delta_{\tau_{i}} \pi_{i}^{l, j+1}(x)-L_{h}^{p} \pi_{i}^{l, j+1}(x)$

$$
\begin{array}{r}
=K_{i}\left(h^{2}+\tau_{i}+\xi_{i}^{l, j}(x)\right), \quad x \in g_{i}^{l} \backslash \partial g_{i}^{l}, \\
i=1, \ldots, M,
\end{array}
$$

(d) $\quad d\left(C_{i}^{l, j}(x)\right) \delta_{\tau_{i}} \pi_{i}^{l, j+1}(x)-L_{h}^{p} \pi_{i}^{l, j+1}(x)$

$$
\begin{array}{r}
=K_{i}\left(h+\tau_{i}+\frac{\tau_{0}^{2}}{h^{2}}+\xi_{i}^{l, j}(x)\right), \quad x \in \partial g_{i}^{l}, \\
i=1, \ldots, M .
\end{array}
$$

Using (27)(b), we can get

$$
\begin{aligned}
\pi_{0}^{l+1}(x)= & \pi_{0}^{l}(x)+\frac{\tau_{0}}{d\left(C_{0}^{l}(x)\right)} L_{h}^{p} \pi_{0}^{l+1}(x) \\
& +K_{0} \tau_{0}\left(h^{2}+\tau_{0}+\xi_{0}^{l}(x)\right)
\end{aligned}
$$

Then using the maximum principle

$$
\begin{aligned}
\max _{x}\left|\pi_{0}^{l+1}(x)\right| \leq & \max _{x}\left|\pi_{0}^{l}(x)\right| \\
& +K_{0} \tau_{0}\left(h^{2}+\tau_{0}+\max _{x}\left|\xi_{0}^{l}(x)\right|\right) .
\end{aligned}
$$

With the method of recursion and noticing that $\pi_{0}^{0}(x)=0$, we obtain the error estimate:

$$
\max _{x}\left|\pi_{0}^{l+1}(x)\right| \leq K_{0} \tau_{0}\left(h^{2}+\tau_{0}+\sum_{k=0}^{l} \max _{x}\left|\xi_{0}^{k}(x)\right|\right) .
$$

Similarly using $(27)(c)$ and (27)(d),

$$
\begin{aligned}
& \max _{x}\left|\pi_{i}^{l, j+1}(x)\right| \leq K_{i} \tau_{i}\left(h^{2}+\tau_{i}+\sum_{k=0}^{j} \max _{x}\left|\xi_{i}^{l, k}(x)\right|\right), \\
& \max _{x}\left|\pi_{i}^{l, j+1}(x)\right| \leq K_{i} \tau_{i}\left(h+\tau_{i}+\frac{\tau_{0}^{2}}{h^{2}}+\sum_{k=0}^{j} \max _{x}\left|\xi_{i}^{l, k}(x)\right|\right) .
\end{aligned}
$$

Then we consider the saturation equation. Suppose that

$$
\left|U_{m, 0}^{l}\right| \leq \bar{U}, \quad\left|U_{m, i}^{l, j}\right| \leq \bar{U}, \quad m=1,2, h \longrightarrow 0,
$$

where $\bar{U}$ is a positive constant. In the end of error analysis, we will prove the supposition (32). Under the supposition (32), we can prove the discretizations (23) satisfy the following property.

Theorem 1. The finite difference schemes (23) comply with the requirements of the maximum principle and the difference operator $L_{h}^{c}$ is coercive in $H_{0}^{1}$, that is, $\exists \mu>0$ such that

$$
-\left(L_{h}^{c} \varphi, \varphi\right) \geq \mu\|\varphi\|_{1, \omega}^{2}, \quad \forall \varphi \in H_{0}^{1}(\omega) .
$$

Considering

$$
\begin{array}{r}
u_{m, 0}^{l}-U_{m, 0}^{l}=K_{0}\left(\xi_{0}^{l}+\nabla_{h} \pi_{0}^{l}+h^{2}\right), \\
u_{m, i}^{l, j}-U_{m, i}^{l, j}=K_{i}\left(\xi_{i}^{l, j}+\nabla_{h} \pi_{i}^{l, j}+h^{2}\right), \\
m=1,2, \quad i=1,2, \ldots, M,
\end{array}
$$

and using (1)(c), (23), we can get the error equation of the saturation equation

$$
\begin{gathered}
\xi(x, t)=0, \quad x \in \partial \Omega, \\
\phi(x) \delta_{\tau_{0}} \xi_{0}^{l+1}-L_{h}^{c} \xi_{0}^{l+1}+\frac{b\left(C_{0}^{l+1}(x)\right)}{d\left(C_{0}^{l+1}(x)\right)} L_{h}^{p} \pi_{0}^{l+1} \\
=K_{0}\left(\tau_{0}+h^{2}+\xi^{l+1}+\nabla_{h} \pi^{l+1}\right), \quad x \in g_{0}^{l}, \\
\phi(x) \delta_{\tau_{i}} \xi_{i}^{l, j+1}-L_{h}^{c} \xi_{i}^{l, j+1}+\frac{b\left(C_{i}^{l, j+1}(x)\right)}{d\left(C_{i}^{l, j+1}(x)\right)} L_{h}^{p} \pi_{i}^{l, j+1} \\
=K_{i}\left(\tau_{i}+h^{2}+\xi_{i}^{l, j+1}+\nabla_{h} \pi_{i}^{l, j+1}\right), \quad x \in g_{i}^{l} \backslash \partial g_{i}^{l}, \\
\phi(x) \delta_{\tau_{i}} \xi_{i}^{l, j+1}-L_{h}^{c} \xi_{i}^{l, j+1}+\frac{b\left(C_{i}^{l, j+1}(x)\right)}{d\left(C_{i}^{l, j+1}(x)\right)} L_{h}^{p} \pi_{i}^{l, j+1}, \ldots, M, \\
=K_{i}\left(\tau_{i}+h+\frac{\tau_{0}^{2}}{h^{2}}+\xi_{i}^{l, j+1}+\nabla_{h} \pi_{i}^{l, j+1}\right), \quad x \in \partial g_{i}^{l},
\end{gathered}
$$

We need an induction hypothesis. Let $\tau_{0}=O(h)$, we assume that

$$
\begin{gathered}
\left|\xi_{0}^{l}\right|=O\left(|\log h|^{1 / 2} h\right), \\
\left|\xi_{i}^{l, j}\right|=O\left(|\log h|^{1 / 2} h\right), \quad h \longrightarrow 0,
\end{gathered}
$$

for $0 \leq l \leq \bar{n}, 0 \leq j \leq \bar{n}$. When $\bar{n}=0$, we can obtain (36) by using (4) and (23). At the end of error analysis, we will prove (36) for $l=\bar{n}+1, j=\bar{n}+1$. 
From error estimates of the pressure equation (30) and (31) and the induction hypothesis (36), we have

$$
\begin{gathered}
\xi(x, t)=0, \quad x \in \partial \Omega, \\
\phi(x) \delta_{\tau_{0}} \xi_{0}^{l+1}-L_{h}^{c} \xi_{0}^{l+1} \\
=K_{0}\left(\tau_{0}+h^{2}+|\log h|^{1 / 2} h\right), \quad x \in g_{0}^{l}, \\
\phi(x) \delta_{\tau_{i}} \xi_{i}^{l, j+1}-L_{h}^{c} \xi_{i}^{l, j+1} \\
=K_{i}\left(\tau_{i}+h^{2}+|\log h|^{1 / 2} h\right), \quad x \in g_{i}^{l} \backslash \partial g_{i}^{l}, \\
\phi(x) \delta_{\tau_{i}} \xi_{i}^{l, j+1}-L_{h}^{c} \xi_{i}^{l, j+1} \quad i=1, \ldots, M, \\
=K_{i}\left(\tau_{i}+h+\frac{\tau_{0}^{2}}{h^{2}}+|\log h|^{1 / 2} h\right), \\
x \in \partial g_{i}^{l}, i=1, \ldots, M .
\end{gathered}
$$

In order to get an estimate for the error $\xi(x, t)$, we need two types of auxiliary functions $\psi_{i}$ and $\varphi_{i}$. The grid functions $\left\{\psi_{i}(x)\right\}_{i=0}^{M}$ and $\left\{\varphi_{i}(x)\right\}_{i=1}^{M}$, respectively, satisfy

$$
\begin{array}{ll}
-L_{h}^{c} \psi_{i}(x)=\chi_{i}(x), & \left.\psi_{i}(x)\right|_{\partial \Omega}=0, \\
-L_{h}^{c} \varphi_{i}(x)=\alpha_{i}(x), & \left.\varphi_{i}(x)\right|_{\partial \Omega}=0,
\end{array}
$$

where $\chi_{i}(x)$ is the characteristic function of $\omega_{i} \backslash \gamma_{i}, \chi_{0}(x)$ is the characteristic function of $\omega_{0}$, and $\alpha_{i}(x)$ is the characteristic function of $\gamma_{i}$. On condition that $L_{h}^{c}$ is coercive in $H_{0}^{1}$, Ewing et al. have proved $\psi_{i}$ and $\varphi_{i}$ satisfy the following lemma [6].

Lemma 2. $\left\{\psi_{i}(x)\right\}_{i=0}^{M}$ and $\left\{\varphi_{i}(x)\right\}_{i=1}^{M}$ exist and are nonnegative, and the following estimates hold:

$$
\begin{aligned}
& \max _{\omega} \psi_{i}(x) \leq C|\log h|^{1 / 2}, \\
& \max _{\omega} \varphi_{i}(x) \leq C h|\log h|^{1 / 2} .
\end{aligned}
$$

Theorem 3. Let the exact solutions $c(x, t)$ of (1) satisfy the condition (6), then the discretization scheme (23) is stable, and if $\tau_{0}=O(h)$ the following estimate for the error holds:

$$
\begin{aligned}
\max _{g}|\xi| \leq|\log h|^{1 / 2} \sum_{i=0}^{M}\{ & C_{i}\left(\tau_{i}+h^{2}+|\log h|^{1 / 2} h\right) \\
& \left.+I_{i}\left(h \tau_{i}+\frac{\tau_{0}^{2}}{h}+|\log h|^{1 / 2} h^{2}\right)\right\} .
\end{aligned}
$$

Proof. Define

$$
\begin{aligned}
\eta(x)= & \sum_{i=0}^{M} \psi_{i}(x) C_{i}\left(\tau_{i}+h^{2}+|\log h|^{1 / 2} h\right) \\
& +\sum_{i=1}^{M} \varphi_{i}(x) I_{i}\left(\tau_{i}+h+\frac{\tau_{0}^{2}}{h^{2}}+|\log h|^{1 / 2} h\right)
\end{aligned}
$$

where $C_{i}=\max _{g_{i}^{l} \backslash \partial g_{i}^{l}}\left|K_{i}(x, t)\right|, I_{i}=\max _{\partial g_{i}^{l}}\left|K_{i}(x, t)\right|$. Using induction over $l$, it easy to observe that

$$
\begin{array}{r}
\phi(x) \delta_{\tau_{0}}\left(\eta(x)-\xi^{l+1}(x)\right)-L_{h}^{c}\left(\eta(x)-\xi^{l+1}(x)\right) \geq 0, \\
x \in g_{0}^{l}, \\
\phi(x) \delta_{\tau_{i}}\left(\eta(x)-\xi_{i}^{l, j+1}(x)\right)-L_{h}^{c}\left(\eta(x)-\xi_{i}^{l, j+1}(x)\right) \geq 0, \\
x \in g_{i}^{l}, i=1, \ldots, M .
\end{array}
$$

Moreover,

$$
\left.(\eta(x)-\xi(x, t))\right|_{\partial \Omega} \geq 0, \quad \forall t \geq 0 .
$$

Using the maximum principle, it follows that

$$
\begin{gathered}
\xi_{0}^{l} \leq \eta(x), \quad x \in \omega_{0}, \\
\xi_{i}^{l, j}(x) \leq \eta(x), \quad x \in \omega_{i}, \quad i=1, \ldots, M, \\
j=1,2, \ldots, m_{i} .
\end{gathered}
$$

Similarly,

$$
\begin{aligned}
-\xi_{0}^{l} \leq \eta(x), \quad x \in \omega_{0}, & \\
-\xi_{i}^{l, j}(x) \leq \eta(x), \quad x \in \omega_{i}, & i=1, \ldots, M, \\
j & =1,2, \ldots, m_{i} .
\end{aligned}
$$

Therefore,

$$
\max _{g}|\xi| \leq \eta(x), \quad x \in \omega_{i}, i=0, \ldots, M .
$$

Then in view of (39), we conclude (40).

It remains to check (32) and the induction hypothesis (36) for $l=\bar{n}+1$. From $\tau_{0}=O(h)$ and the error estimate (40), it is easy to obtain (36). Then using (30) and (31), (34), and (40), we can obtain the supposition (32). The proof of Theorem 3 is complete.

\section{Numerical Results}

We consider a system of coupled partial differential equations:

$$
\begin{gathered}
\frac{\partial p}{\partial t}+\frac{\partial u}{\partial x}=q(x, t), \quad(x, t) \in \Omega \times J, \\
u=-\frac{\partial p}{\partial x}, \quad(x, t) \in \Omega \times J, \\
\frac{\partial c}{\partial t}+\frac{\partial p}{\partial t}+u \cdot \frac{\partial c}{\partial x}-D(x) \frac{\partial^{2} c}{\partial x^{2}} \\
=f(c, x, t), \quad(x, t) \in \Omega \times J, \\
c(x, 0)=c_{0}(x), \quad x \in \Omega, \\
c(0, t)=c_{l}(t), \quad c(1, t)=c_{r}(t), \quad t \in J,
\end{gathered}
$$


TABLE 1: Error estimate in maximum norm when $D(x)=1$.

\begin{tabular}{|c|c|c|c|c|c|c|}
\hline \multirow{2}{*}{$m$} & \multicolumn{3}{|c|}{$t=0.5$} & \multicolumn{3}{|c|}{$t=1$} \\
\hline & Computational cost & $\gamma$ & Reduction & Computational cost & $\gamma$ & Reduction \\
\hline 1 & 0.1560 & 0.0159 & & 0.3130 & 0.0356 & \\
\hline 2 & 0.5780 & 0.0062 & 2.56 & 1.1560 & 0.0137 & 2.60 \\
\hline 4 & 2.3440 & 0.0026 & 2.38 & 4.6080 & 0.0058 & 2.36 \\
\hline 8 & 9.2810 & 0.0012 & 2.17 & 18.6800 & 0.0026 & 2.23 \\
\hline
\end{tabular}

TABLE 2: Error estimate in maximum norm when $D(x)=x$.

\begin{tabular}{|c|c|c|c|c|c|c|}
\hline \multirow{2}{*}{$m$} & \multicolumn{3}{|c|}{$t=0.5$} & \multicolumn{3}{|c|}{$t=1$} \\
\hline & Computational cost & $\gamma$ & Reduction & Computational cost & $\gamma$ & Reduction \\
\hline 1 & 0.1710 & 0.0535 & & 0.3270 & 0.1342 & \\
\hline 2 & 0.5920 & 0.0254 & 2.11 & 1.1680 & 0.0641 & 2.10 \\
\hline 4 & 2.3840 & 0.0124 & 2.05 & 4.6590 & 0.0313 & 2.05 \\
\hline 8 & 9.8810 & 0.0061 & 2.03 & 19.7440 & 0.0154 & 2.03 \\
\hline
\end{tabular}

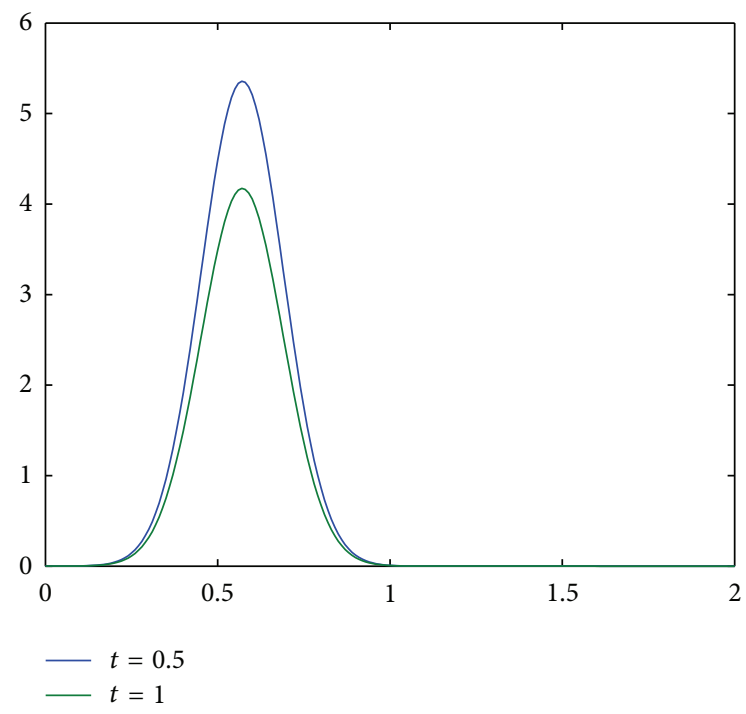

Figure 2: The exact solution $p$.

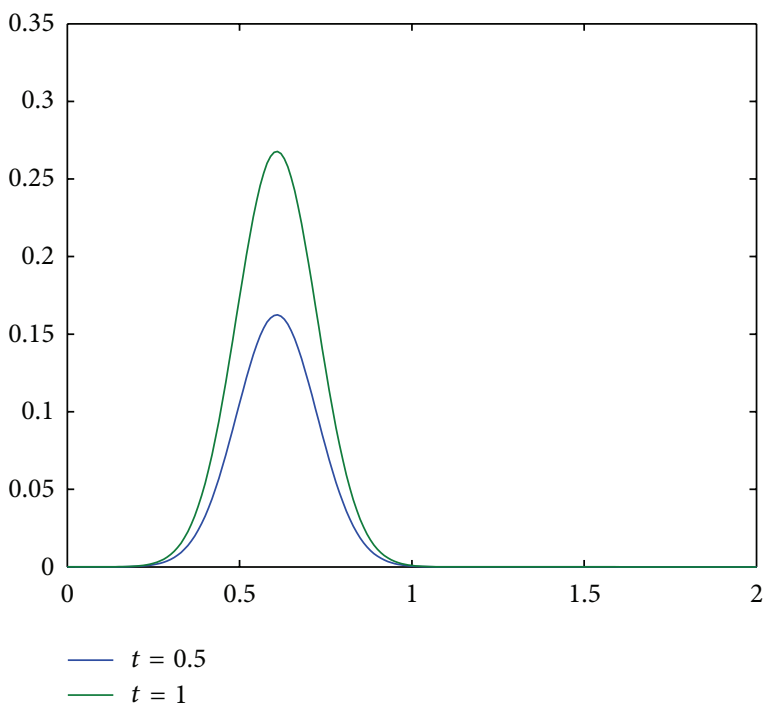

FIgURE 3: The exact solution $c$. where $\Omega=[0,2]^{2}, J=[0, T]$. The following functions are used as exact solutions of (47):

$$
\begin{aligned}
& p=\exp \left(t-t^{2}\right) \exp \left(-35 x^{2}+40 x-10\right) \\
& c=\exp (t) \exp \left(-37 x^{2}+45 x-16\right)
\end{aligned}
$$

When $t=0.5$ and $t=1$, exact solutions of (47) are shown in Figures 2 and 3. Specific forms of $c_{0}, c_{l}, c_{r}, q$, and $f$ are derived by exact solutions.

From Figures 2 and 3, we can see exact solutions of (47) possess highly localized properties in $[0.2,1]^{2}$. First, $\Omega$ is discretized using a regular grid. Let the space-step $h=1 / 160$ and the time-step $\tau_{0}=h$. Then choose the subregion $\Omega_{1}=[0,1.3]^{2}$, which is refined in time. Let the discretization parameters $\tau^{\prime}=\tau_{0} / m$ in the refined region $\Omega_{1}$, where $m$ is a positive integer. We denote by $C$ the numerical approximation to $c$ obtained by (23). And let the error estimate in maximum norm $\gamma=\max _{g}|c-C|$.

Example 4. Let $D(x)=1$. Choosing $m=1,2,4,8$, computational results obtained by (23) are shown in Figures 4 and 5 and Table 1.

Example 5. Let $D(x)=x$. Choosing $m=1,2,4,8$, computational results obtained by (23) are shown in Table 2.

From Figures 4 and 5 and Tables 1 and 2, we can see that numerical results produced by using the local refinement technique are more accurate than those produced without refinement. From Tables 1 and 2, we observe a monotonic improvement of the accuracy in maximum norm when using difference refinement factors $m$. These results are of great importance for the research on numerical simulation of the fluid flow problem and also indicate that the method 


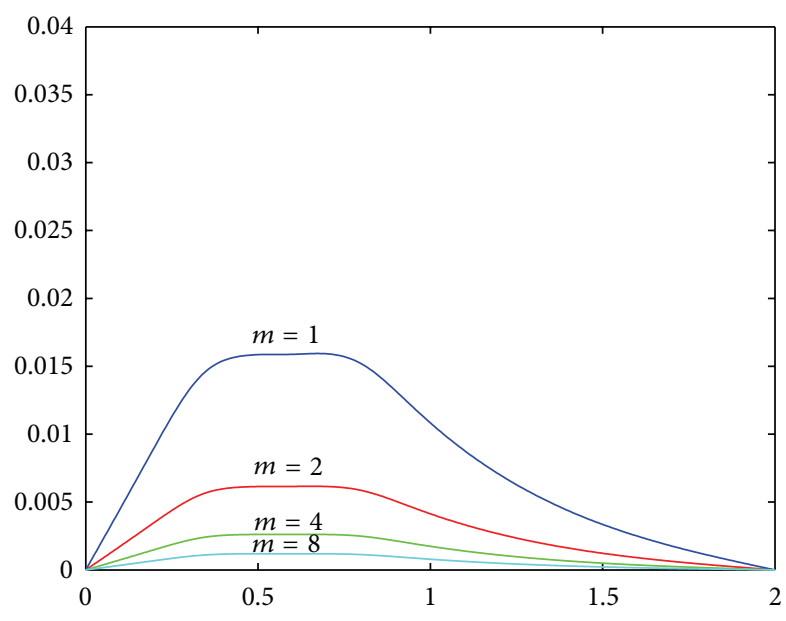

Figure 4: Error $|c-C|$ when $t=0.5$.

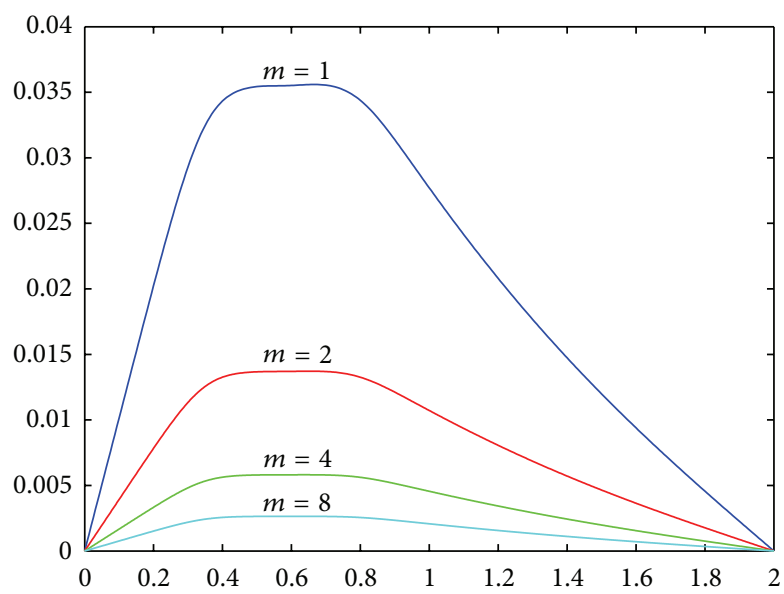

FIgURE 5: Error $|c-C|$ when $t=1$.

proposed in this paper can be widely applied to some application fields, such as energy numerical simulation and environmental science.

\section{Acknowledgments}

The author thanks Professor Yirang Yuan for his valuable constructive suggestions which lead to a significant improvement of this paper. This work is supported in part by the National Natural Science Foundation of China (Grant no. 71071088) and the Natural Science Foundation of Shandong Province of China (Grant no. ZR2011AQ021).

\section{References}

[1] Y. R. Yuan, "Some new progress in the fileds of computational petroleum geology and others," Chinese Journal of Computational Physics, vol. 20, no. 4, pp. 283-290, 2003.

[2] R. E. Ewing, R. D. Lazarov, and P. S. Vassilevski, "Local refinement techniques for elliptic problems on cell-centered grids. I. Error analysis," Mathematics of Computation, vol. 56, no. 194, pp. 437-461, 1991.

[3] Z. Q. Cai, J. Mandel, and S. F. McCormick, "The finite volume element method for diffusion equations on general triangulations," SIAM Journal on Numerical Analysis, vol. 28, no. 2, pp. 392-402, 1991.

[4] Z. Q. Cai and S. F. McCormick, "On the accuracy of the finite volume element method for diffusion equations on composite grids," SIAM Journal on Numerical Analysis, vol. 27, no. 3, pp. 636-655, 1990.

[5] R. E. Ewing, R. D. Lazarov, and P. S. Vassilevski, "Finite difference schemes on grids with local refinement in time and space for parabolic problems. I. Derivation, stability, and error analysis," Computing, vol. 45, no. 3, pp. 193-215, 1990.

[6] R. E. Ewing, R. D. Lazarov, and A. T. Vassilev, "Finite difference scheme for parabolic problems on composite grids with refinement in time and space," SIAM Journal on Numerical Analysis, vol. 31, no. 6, pp. 1605-1622, 1994.

[7] W. Liu and Y. R. Yuan, "Finite difference schemes for twodimensional miscible displacement flow in porous media on composite triangular grids," Computers \& Mathematics with Applications, vol. 55, no. 3, pp. 470-484, 2008.

[8] W. Liu and Y. R. Yuan, "A finite difference scheme for twodimensional semiconductor device of heat conduction on composite triangular grids," Applied Mathematics and Computation, vol. 218, no. 11, pp. 6458-6468, 2012.

[9] J. Douglas, Jr. and J. E. Roberts, "Numerical methods for a model for compressible miscible displacement in porous media," Mathematics of Computation, vol. 41, no. 164, pp. 441459, 1983.

[10] R. Yi Yuan, "Time stepping along charcteristics for the finite element approximation of compressible miscible displacement in porous media," Mathematica Numerica Sinica, vol. 14, no. 4, pp. 385-406, 1992.

[11] J. M. Yang and Y. P. Chen, "A priori error analysis of a discontinuous Galerkin approximation for a kind of compressible miscible displacement problems," Science China, Mathematics, vol. 53, no. 10, pp. 2679-2696, 2010. 


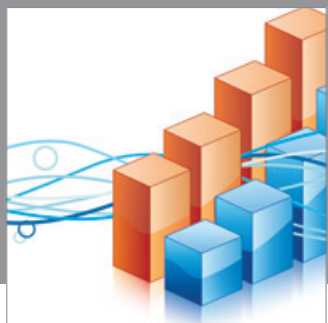

Advances in

Operations Research

mansans

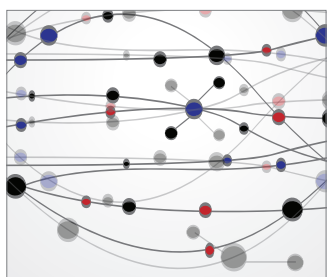

The Scientific World Journal
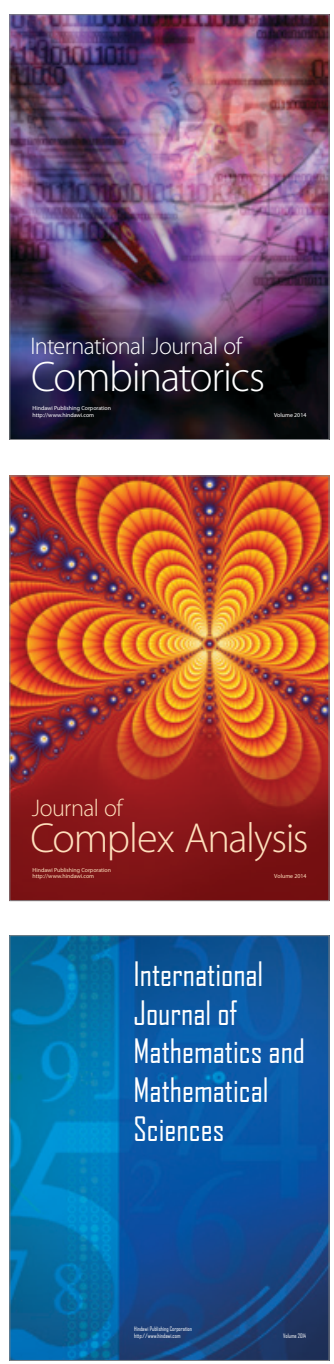
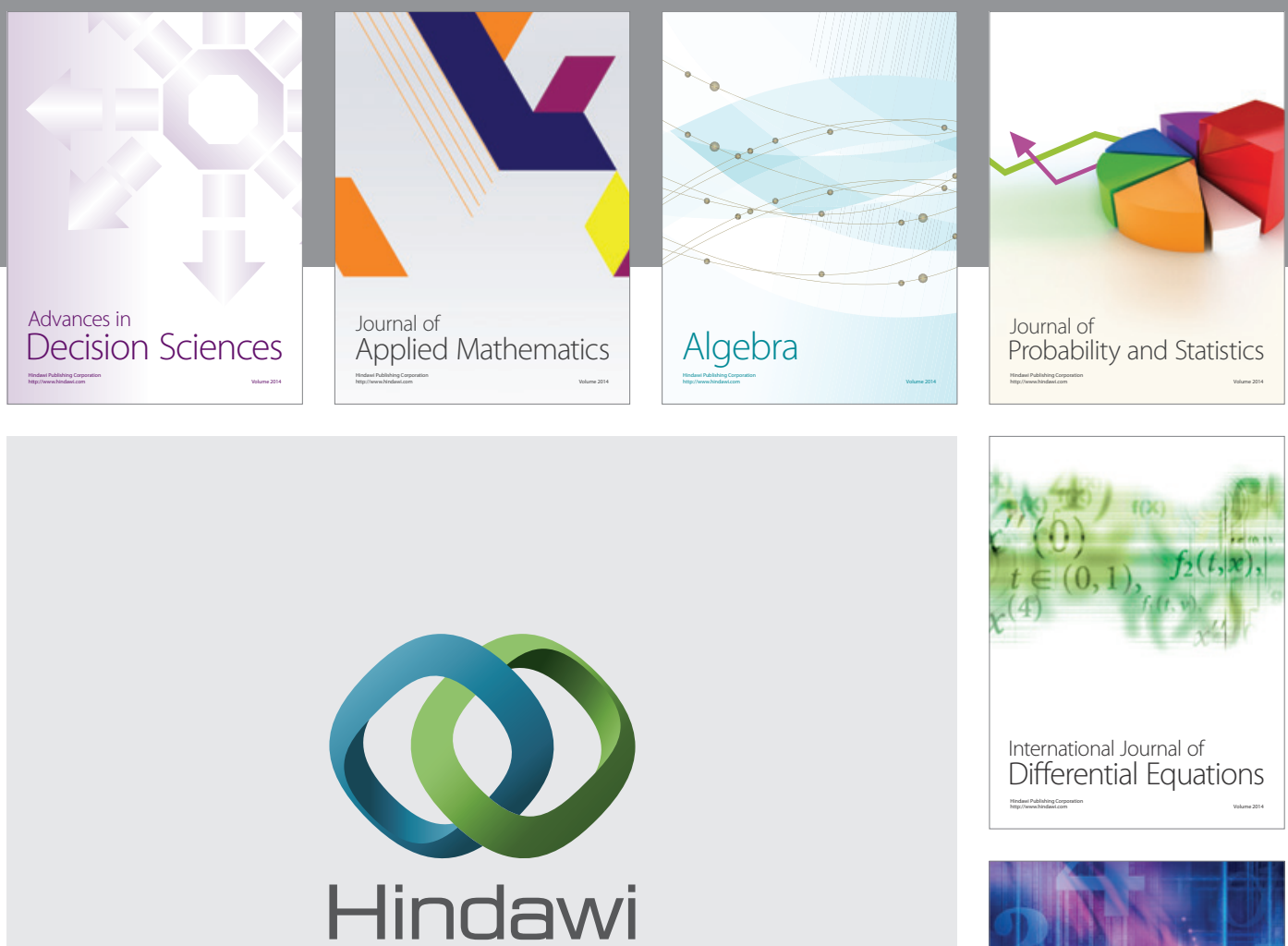

Submit your manuscripts at http://www.hindawi.com
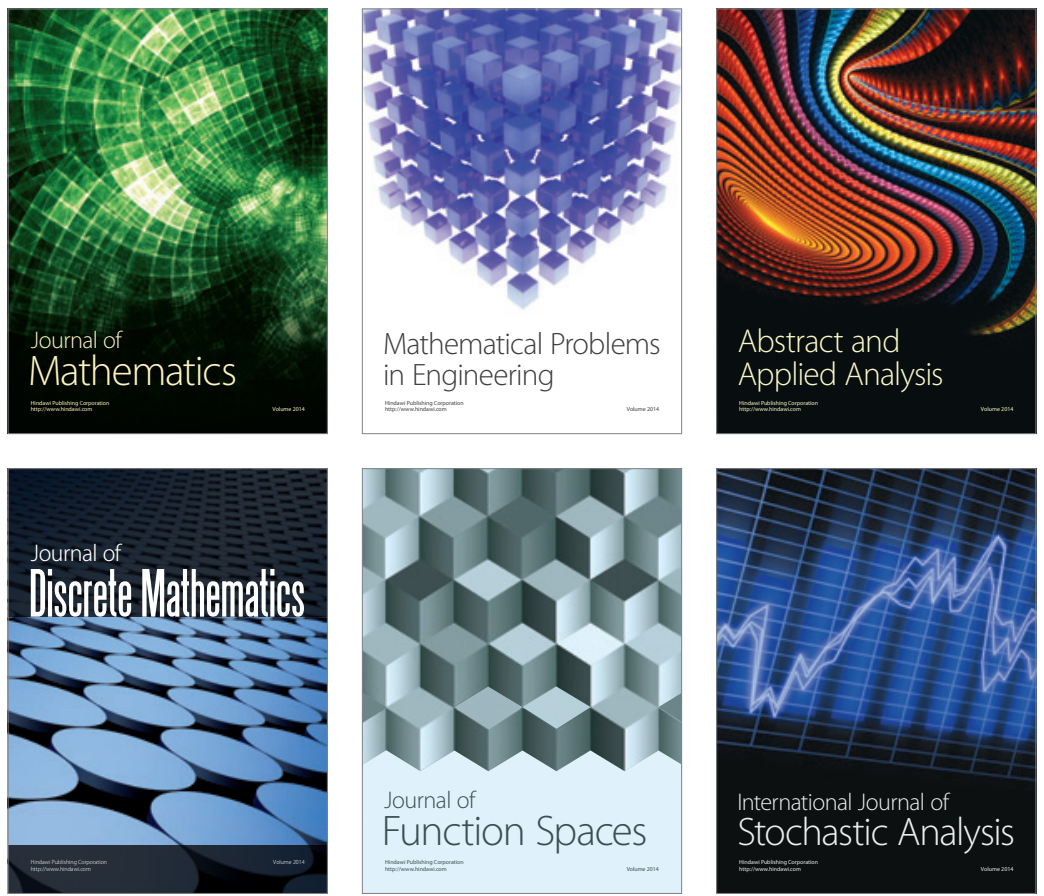

Journal of

Function Spaces

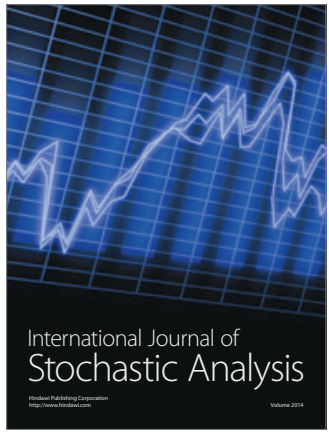

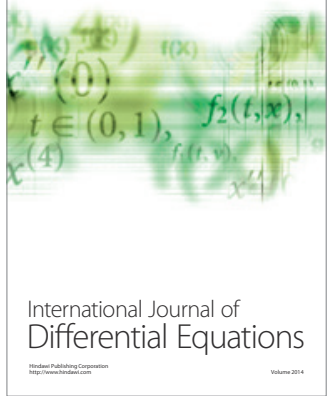
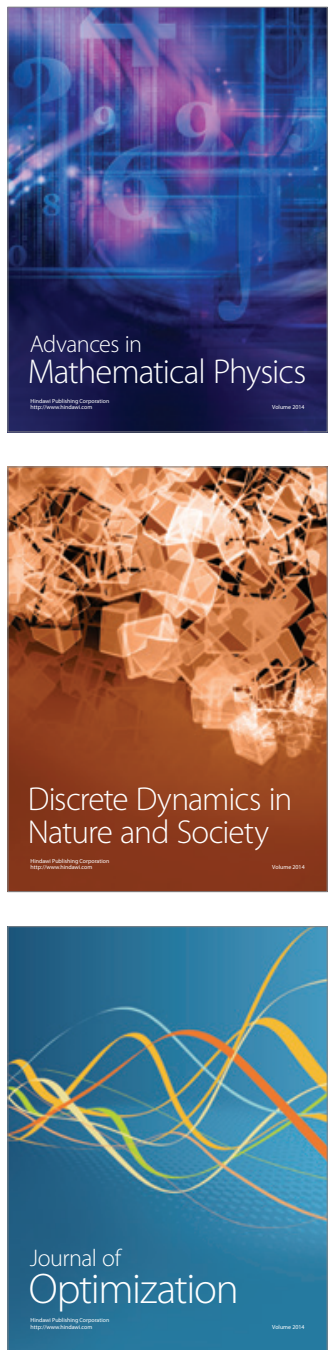